

\title{
Human Placental Arterial Distensibility, Birth Weight, and Body Size Are Positively Related to Fetal Homocysteine Concentration
}

\author{
Stephen W. D'Souza, FRCPCH ${ }^{1}$, Nita Solanky, PhD ${ }^{1}$, Jane Guarino, SRN ${ }^{1}$, Stuart Moat, FRC Path ${ }^{2}$, \\ Colin P. Sibley, DSc ${ }^{1}$, Michael Taggart, $\mathrm{PhD}^{3}$, and Jocelyn D. Glazier, $\mathrm{PhD}^{1}$
}

\footnotetext{
${ }^{1}$ Maternal and Fetal Health Research Centre, Division of Developmental Biology and Medicine, School of Medical Sciences, Faculty of Biology, Medicine and Health, University of Manchester, Manchester Academic Health Science Centre, St Mary's Hospital, Manchester, UK

${ }^{2}$ Department of Medical Biochemistry and Immunology, University Hospital of Wales and Cardiff School of Medicine, Cardiff University, Heath Park, Cardiff, United Kingdom

3 Institute of Genetic Medicine, International Centre for Life, Newcastle University, Newcastle, United Kingdom
}

\section{$\underline{\text { Keywords }}$}

Chorionic Plate Arteries, Folate, Neonatal Blood Pressure, Vascular Function, Vitamin $\mathrm{B}_{12}$

\begin{abstract}
Methionine demethylation during metabolism generates homocysteine ( $\mathrm{Hcy}$ ) and its remethylation requires folate and cobalamin. Elevated Hcy concentrations are associated with vascular-related complications of pregnancy, including increased vascular stiffness, predictive of clinical vascular disease. Maternal and fetal total Hcy (tHcy) concentrations are positively related, yet the influence of Hcy on fetoplacental vascular function in normal pregnancy has not been examined. We hypothesized that Hcy alters fetoplacental vascular characteristics with influences on fetal growth outcomes. We investigated (1) placental chorionic plate artery distensibility and neonatal blood pressure in relation to umbilical plasma tHcy; (2) relationships between cord venous (CV) and cord arterial (CA) plasma tHcy, folate, and cobalamin concentrations; and (3) tHcy associations with birth weight and anthropometric measurements of body size as indices of fetal growth in normal pregnancies with appropriate weight-for-gestational age newborns. Maternal plasma tHcy, folate, and cobalamin concentrations were consistent with published data. Placental chorionic plate artery distensibility index ( $\beta$; measure of vessel stiffness) was inversely related to CA tHcy, yet neonatal blood pressure was not significantly affected. CV and CA tHcy concentrations were positively related and CV tHcy negatively related to $\mathrm{CV}$ cobalamin but not folate. $\mathrm{CV}$ tHcy concentration positively related to birth weight, corrected birth weight per-centile, length, head circumference, and mid-arm circumference of newborns. CV cobalamin was inversely related to fetal growth indices but not to folate concentration. Our study demonstrates a potential relationship between fetal tHcy and placental artery distensibility, placing clinical relevance to cobalamin in influencing Hcy concentration and maintaining low vascular resistance to facilitate nutrient exchange favorable to fetal growth.
\end{abstract}




\section{$\underline{\text { Introduction }}$}

Homocysteine (Hcy) arises from the metabolism of methionine (Figure 1), which crucially generates the methyl donor S-adenosylmethionine (SAM) required for cellular methylation processes including DNA, RNA, protein, and phospholipid methylation. ${ }^{1}$ Methyl donation from SAM to cellular acceptors generates S-adenosylhomocysteine (SAH). The efficient metabolism of SAH (by SAH hydrolase catalysis to produce adenosine and Hcy) is essential, as SAH inhibits the activity of SAMdependent methyltransferases involved in a variety of methylation reactions. Homocysteine can undergo remethylation back to methionine (Figure 1), and this conversion requires folate and cobalamin (Cbl) catalyzed by the action of methionine synthase. ${ }^{1,2}$ Importantly, this is the main pathway for the metabolic disposal of Hcy in human placenta. ${ }^{1}$ Hence, suboptimal folate or $\mathrm{Cbl}$ status, or diminished Hcy-metabolizing enzyme activity, can lead to dysregulated Hcy biosynthesis and elevated plasma total Hcy (tHcy) concentration. ${ }^{3-5}$ Human pregnancy is a period of increased folate and $\mathrm{Cbl}$ demand ${ }^{6}$ and disturbances in $\mathrm{Hcy}$-folate-Cbl metabolism associate with clinical complications affecting uteroplacental vascular function with impacts on fetal and neonatal development. ${ }^{7-10}$

Several studies have demonstrated that maternal tHcy concentration is higher in infants of low birth weight than normal controls, ${ }^{8,11-14}$ and a meta-analysis has revealed a $25 \%$ increased risk of having a small-for-gestational age infant when maternal tHcy concentration exceeded the 90th percentile. ${ }^{11}$ However, observations are inconsistent; other studies report that low birth weight is not associated with raised maternal tHcy concentration ${ }^{15,16}$ or demonstrate both negative ${ }^{17}$ and positive ${ }^{18}$ associations between maternal tHcy concentration and birth weight. A consistent interpretation of these data is challenging, as effects of tHcy on birth weight per se may not be easily distinguished from effects of accompanying suboptimal B vitamin status that would generate tHcy through metabolic-interdependent pathways (Figure 1). A more informative situation is presented when availability of maternal folate and $\mathrm{Cbl}$ is optimal, and therefore, fetal provision of these is not limiting, in determining the relationship between tHcy and fetal growth outcomes.

Raised tHcy is an independent risk factor for vascular disease ${ }^{19,20}$; it associates with several aspects of impaired vascular function including endothelial dysfunction, ${ }^{4,21-23}$ enhanced smooth muscle proliferation, ${ }^{24}$ stimulated collagen biosynthesis, ${ }^{25,26}$ and diminished vascular elasticity, ${ }^{27}$ leading to increased arterial stiffness. However, much remains to be understood about the potential relationship between tHcy and indices of arterial stiffness in the fetoplacental circulation in normal pregnancy.

We hypothesized that as fetal Hcy increases, distensible properties of arteries in the fetoplacental circulation would be impaired, leading to an increased vascular resistance, which in turn could influence fetal growth and vascular indices in the neonate. Hence, a primary objective of this study was to investigate whether cord plasma tHcy concentrations influence the distensibility characteristics of small chorionic plate arteries (CPAs) of human placenta, selected for their 
relative ease of accessibility and a likely site of resistance in the fetoplacental circulation. ${ }^{28-30}$ Additionally, we examined whether neonatal blood pressure (BP), as an index of vascular resistance, was influenced by fetal tHcy concentration and determined the relationships between fetal tHcy in umbilical cord venous (CV) and cord arterial (CA) plasma to birth weight and anthropometric measures of neonatal body proportions.

\section{Materials and Methods}

\section{Mothers}

All women (European, white Caucasian) were in good health and gave signed informed consent at St Mary's Hospital, Manchester. Ethical approval was granted by the Central Manchester Local Research Ethics Committee (06/Q1407/25) with University of Manchester Committee on the Ethics of Research on Human Beings endorsement (06040). Obstetric records confirmed normal uncomplicated pregnancies. Maternal characteristics were obtained at the first antenatal clinic attendance (10-12 weeks). Gestational age was defined as completed weeks of gestation, using menstrual dates and confirmed by ultrasound dating. The women reported routine use of folic acid supplements ( $0.4 \mathrm{mg} / \mathrm{d}, 4-8$ weeks duration in early pregnancy), a non-vegetarian diet, and not smoking cigarettes.

\section{Newborn Infants, Anthropometric Measurements, and BP}

All infants were in good condition at birth (Apgar scores 9 or 10 at 1 minute). Percentiles for birth weight were determined using national growth standards (UK-WHO Growth Charts for Children aged 0-4 years, Child Growth Foundation, UK). On routine clinical examination, no medical condition or congenital abnormality was recorded. The same research nurse measured birth weight using a digital baby scale (Seca 384, Germany) and anthropometric indices. Occipital-frontal head circumference and mid-arm circumference with the left arm flexed to 90 at the elbow were measured using a paper measuring tape. Length was measured using a Harpenden Neonatometer (Holtain Limited, United Kingdom). Blood pressure was measured using a Dinamap Pro 100 automated oscillometric machine with an appropriate BP cuff (GE Medical Systems, Information Technologies, Freiburg, Germany) at 24 to 48 hours following birth. ${ }^{31}$

\section{Plasma tHcy, Folate, and Cbl Analysis}

Non-fasting maternal blood was obtained from the antecubital vein at antenatal clinic (10-12 weeks). The CV and CA blood was obtained at delivery after clamping the umbilical cord. Maternal and cord blood was collected into tubes with either potassium EDTA or lithium heparin as anticoagulant, placed on ice, and centrifuged at $1000 \mathrm{~g}$ for 10 minutes. Separated plasma was stored at $80 \mathrm{C}$ and transferred for analysis (University Hospital of Wales, Cardiff). Plasma tHcy was measured by high-performance liquid chromatography and fluorometric detection following reduction, deproteinization, and derivatization with the fluorophore SBDF (ammonium 7-fluorobenzo2-oxa-1,3-diazole-4-sullfonate). ${ }^{32}$ This assay was standardized using National Institute of Standards 
and Technology Standard Reference Material. Plasma folate and Cbl were measured by competitive protein-binding assays on an Elecys 2010 analyzer (Roche Diagnostics, Burgess Hill, West Sussex, UK).

\section{Pressure Myography Studies on CPAs}

Pressure myography studies were performed to measure the passive mechanical wall properties of the CPAs. ${ }^{30,33}$ Placentas were obtained within 30 minutes of delivery, and CPAs ( $<300 \mathrm{~mm}$ diameter) were isolated and mounted onto 2 glass cannulae connected to a pressure servo unit to regulate transmural pressure (Living Systems Instrumentation, Burlington, Vermont). The arteriography chamber was perfused with calcium-free physiological solution (with the following composition [in mmol/L]: $\mathrm{NaCl} 119, \mathrm{NaHCO}_{3} 25, \mathrm{KCl} 4.69, \mathrm{MgSO}_{4}$ 2.4, EGTA 2, $\mathrm{KH}_{2} \mathrm{PO}_{4}$ 1.18, glucose 6.05, EDTA 0.034, $\mathrm{pH} 7.4$; gassed with $5 \% \mathrm{O}_{2}$ and $5 \% \mathrm{CO}_{2}$ ) and maintained at $37^{\circ} \mathrm{C}$. Intraluminal pressure was increased stepwise from 3 to $120 \mathrm{~mm} \mathrm{Hg}$. Vessels were continuously analyzed by video dimension analysis, and arterial lumen diameter and wall thicknesses were measured at 3 sites along the vessel length (and averaged). Wall cross-sectional area (CSA) was calculated as $p \succsim r^{0} r^{1} p$, where $r_{0}$ and $r_{1}$ are whole vessel and lumen radii, respectively. These measurements allowed calculation of wall stress and strain. Wall stress $\left(\right.$ dyne $\left./ \mathrm{cm}^{2}\right)$ was calculated as: pressure lumen diameter/(2 wall thickness). Strain was calculated as: increase in lumen diameter from $3 \mathrm{~mm}$ $\mathrm{Hg} /$ diameter at $3 \mathrm{~mm} \mathrm{Hg}$. From plots of the stress-strain relationship $\left(r^{2}>.9\right)$, where stress $=$ $y . e^{\beta . s t r a i n}$, the coefficient of intrinsic vessel stiffness $(b)$ was derived from the gradient.

\section{Data Analysis}

Statistical analyses were carried out with SPSS 11.0 for Windows (IBM SPSS, Portsmouth, Hampshire, UK) or Prism version 6 (GraphPad Software, La Jolla, California). Data are presented in various formats as detailed in the text, with $n=$ number of individual pregnancies. Birth weight was adjusted for maternal height, weight, ethnicity and parity, infant sex, and gestational age to determine corrected birth weight percentile. ${ }^{34}$ Median tHcy, folate, and Cbl values in CV and CA blood were compared using Mann-Whitney $\mathrm{U}$ or Wilcoxon signed rank test as appropriate. Spearman correlation coefficient was used to investigate relationships of CV plasma tHcy, folate, or Cbl with birth weight and anthropometric measurements; relationship of CV and CA plasma tHcy with systolic, diastolic, or mean BP; and relationship between CA plasma tHcy and CPA stiffness index ( $\beta$ ). Linear regression analysis was used to examine the relationship between paired CV and CA tHcy concentration. Kruskal-Wallis test assessed the significance of corrected birth weight percentile relationships to paired CV tHcy and CA tHcy concentrations. $\mathrm{P}<.05$ was considered statistically significant.

\section{$\underline{\text { Results }}$}

\section{Maternal and Infant Characteristics}

Maternal and infant characteristics are shown in Table 1. Birth weights were appropriate for gestational age ( $>25$ th and $<90$ th percentiles), and BP was consistent with reference values. ${ }^{31}$ 
To examine the passive mechanical properties of CPA, vessel dimensional changes in response to varying intraluminal pressures were assessed. The lumen diameter of CPA vessels expanded as intraluminal pressure was raised incrementally, with the most dynamic increase in lumen diameter occurring up to an applied pressure of $30 \mathrm{~mm} \mathrm{Hg}$ (Figure 2A). Wall thickness exhibited a gradual decline over the same range of applied pressures (Figure 2B), with a gradual increase in wall CSA (Figure 2C). All CPA vessels were responsive to pressure changes, confirming preservation of vascular integrity. As expected, the stress-strain curve generated by CPA demonstrated an exponential relationship (Figure 2D), and from individual stress-strain relationship curves, the coefficient of vessel stiffness (b) was derived. Figure 2E shows stratification of the stress-strain relationship curves according to umbilical arterial tHcy concentration quartiles. The CPA vessels of placentas where CA plasma tHcy concentrations were in the lowest quartile demonstrated a left shift relative to those in higher quartiles, suggesting that these CPA vessels were stiffer (less distensible). Conversely, those CPA vessels from placentas with CA tHcy concentrations falling in the highest quartile 1 demonstrated a right shift relative to those in lower quartiles, suggesting these CPA vessels were less stiff with greater vessel 3 or more distensibility. As shown in Figure 2F, values of $b$, the coefficient of vessel stiffness, ranged from 2.2 to 12.2, reflecting a large variability in the distensible properties of CPA vessels of placentas from normal pregnancies. Vessel stiffness in placental CPA was inversely related to plasma tHcy concentration over the concentration range of 4.5 to $10.8 \mathrm{mmol} / \mathrm{L}$ tHcy in CA plasma (Figure 2F), which in vivo would flow through CPA of the fetoplacental circulation. The concentration of tHcy in CA plasma was highly dependent upon the concentration of tHcy in the umbilical venous circulation as CV and CA tHcy concentrations were highly correlated ( $r 21 / 4.94, \mathrm{P}<.001$; Figure 3$)$, in agreement with previous observations. ${ }^{17,35}$ This raises the possibility that $\mathrm{tHcy}$ arising from placental transport and/or metabolism of Hcy and entering the fetal circulation could ultimately influence the distensible properties of placental CPA vessels. Neonatal systolic, diastolic, and mean BP was not significantly associated with either CV or CA tHcy concentration (data not shown).

\section{Plasma tHcy, Folate, and Cbl Concentrations in Maternal and Cord Blood}

Maternal plasma tHcy, folate, and Cbl concentrations (median [quartiles], $\mathrm{n}$ - 23) were 4.6 [3.7, 5.9] $\mathrm{mmol} / \mathrm{L}, 12.4[6.8,19.8] \mathrm{ng} / \mathrm{mL}$, and $208[163,268] \mathrm{ng} / \mathrm{L}$, respectively, consistent with the previous studies. ${ }^{36}$ In a subset of paired cord plasma samples, tHcy concentration was significantly higher in CV than CA plasma $(6.30[5.60,9.95] \mathrm{mmol} / \mathrm{L}$ vs $6.10[4.75,8.60] \mathrm{mmol} / \mathrm{L}, \mathrm{P}=.0006)$ with a similar trend toward significance for $\mathrm{Cbl}(346[241,374] \mathrm{ng} / \mathrm{L}$ vs $285[225,352] \mathrm{ng} / \mathrm{L}, \mathrm{P}<.07)$, while folate concentrations (18.15 [13.10, 38.82] ng/L vs 17.45 [12.45, 31.85] ng/L) were not significantly different $(P=.71 ; n=25$ for all). CV tHcy exhibited a significant negative correlation with $\mathrm{CV} \mathrm{Cbl}(r=$ $.53, P<.005 ; n=27)$ but not CV folate $(r=.31, P=.12 ; n=26)$, suggesting thcy entering the fetal circulation was particularly modulated by $\mathrm{Cbl}$. These associations were not statistically significant in CA plasma.

Relationships of Plasma tHcy, Folate, and Cbl Concentrations in Cord Blood to Birth Weight and Neonatal Anthropometric Indices 
Cord venous tHcy concentration was positively correlated with birth weight, corrected birth weight percentile, neonate length, head circumference, and mid-arm circumference (Table 2). CV Cbl was negatively correlated with birth weight, corrected birth weight percentile, neonate length, and midarm circumference (Table 2). CV folate demonstrated no significant relationships. Stratification of birth weight into percentile ranges revealed that corrected birth weight percentiles were positively associated with a graduated elevation of both CV and CA plasma tHcy concentrations (Figure 4).

\section{Discussion}

This study has revealed novel relationships between CA tHcy concentration and the distensibility characteristics of placental CPA that importantly provide oxygen and nutrients and remove waste products during development and reflect a site of vascular resistance within the fetoplacental circulation. ${ }^{37,38}$ We observed that CPA isolated from placentas of normal, uncomplicated pregnancies exhibited greater variability in their distensible properties as compared to other vessel types, ${ }^{39-42}$ evidenced by the variability in the value of $\beta$, the coefficient of CPA vessel stiffness (Figure 2). This variability may, however, reflect an influence of maternal diet; interdependencies between tHcy, folate, and $\mathrm{Cbl}$ can influence arterial stiffness. ${ }^{43,44}$ The structural characteristics of CPA previously reported include a lack of internal elastic lamina and a dominance of collagen fibers orientated around the smooth muscle cells of the arterial wall. ${ }^{38}$ Whether greater intrinsic vessel stiffness (higher $\beta$ value) is underscored by changes in the content and/or arrangement of extracellular matrix proteins as major determinants of passive vascular mechanical properties ${ }^{45}$ requires further investigation.

Previous studies examining the effects of hyperhomocysteinemia on vascular remodeling have shown an increased deposition of collagen and vessel stiffness leading to increased vascular resistance and ensuing vascular pathology. ${ }^{46}$ However, the novelty of the current study is that it has investigated the relationships between vessel CPA distensibility and "physiological" concentrations of tHcy (as accepted in clinical practice) in normal, uncomplicated pregnancy. We have demonstrated a clear association between these variables in matched samples, with a lower intrinsic stiffness and greater distensibility of placental CPA vessels found as the tHcy concentration in CA plasma increased (Figure 2). It was notable that the distensibility of isolated placental CPA was related to tHcy concentration in the umbilical arterial circulation (flowing from the fetus to the placenta) over a relatively narrow tHcy concentration range $(4.5-10.8 \mathrm{mmol} / \mathrm{L}$; Figure 2$)$. Variability in CA plasma tHcy concentration is likely to be dictated by that in the umbilical venous circulation (flowing from the placenta to the fetus) based on the strong correlation between umbilical venous and arterial plasma tHcy concentrations (Figure 3). These observations are of interest in raising 2 possibilities: either subtle changes in CA plasma tHcy concentration in the umbilical arterial circulation affects CPA vascular distensibility or greater efflux of Hcy from the placenta to the umbilical venous circulation occurs in placentas with greater CPA vessel distensibility. In this context, it is noteworthy that Hcy can elicit dilation in other vessel types in a dose-dependent manner. ${ }^{47}$ Homocysteine in the umbilical venous circulation could originate either from maternal plasma by placental transport of $\mathrm{tHcy}^{48,49}$ with maternal tHcy concentration predicting tHcy in cord blood, 4,35,36,50,51 or alternatively could be generated by placental metabolism, perhaps reflective of placental methylation capacity. ${ }^{1,49}$ However, there was no apparent influence of tHcy in the fetal circulation on neonatal BP, suggesting that the associations may reflect vessel-specific phenomena. 
We went on to consider how the relationship between tHcy and placental CPA distensibility might impact on fetal growth. A relatively greater CPA distensibility would be consistent with a lower resistance in the fetoplacental circulation, of potential benefit for placental nutrient delivery and fetal growth. Consistent with this concept, we observed positive associations between increasing tHcy concentration in both fetal circulations and birth weight outcomes (as a proxy of growth; Figure 4). The lack of a relationship between fetal folate concentration and birth weight or body size observed here in a folate-supplemented cohort is consistent with the observation of others who also demonstrate a lack of dependency with respect to maternal folate status. ${ }^{12,13,52}$

In our study in normal pregnancy, maternal and cord plasma tHcy, folate, and Cbl concentrations accord well with those from previous larger studies where women routinely used folic acid supplements, ${ }^{36,50}$ providing confidence in the translatability of the findings. Further, the CV-CA tHcy relationships observed here (Figure 3 ) are consistent with the previous studies. ${ }^{17,35}$ Fetal plasma tHcy shows an inverse association with both maternal and fetal folate and $\mathrm{Cbl}$ concentrations, respectively. ${ }^{35,50-53}$ Our demonstration of a significant inverse relationship between tHcy and $\mathrm{Cbl}$ concentrations in CV plasma accords well with these previous observations, $35,36,52,53$ suggesting fetal tHcy concentration is highly modulated by the availability of B-group vitamins. The concept of a regulatory dominance of fetal $\mathrm{Cbl}$ on tHcy concentration $35,36,50,53$ is strengthened here by the reciprocal associations between fetal tHcy and $\mathrm{Cb}$ l with respect to birth weight and anthropometric measurements of neonatal body size (Table 2).

Importantly, our study has several strengths over the previous studies designed to investigate the influence of tHcy on birth weight in that we also performed measurement of anthropometric indices to augment determination of birth weight as a proxy of fetal growth outcome. Additionally, participants were a well-defined cohort of the same ethnicity, and all infants were of appropriate-forgestational age weight. We excluded smokers and selected women who had fetal ultrasound scans to confirm gestational age and who gave birth at 37 to 42 weeks, negating the effects of smoking and gestational age as determinants of birth weight that have associations with tHcy. ${ }^{10,52,54}$ The strength of the association between birth weight and CV tHcy and CV Cbl was explored further by using corrected birth weight percentile, adjusting for maternal height, weight, ethnicity and parity, infant sex, and gestational age. It is notable that our findings are consistent with those of larger populations who have reported a negative association between $\mathrm{Cbl}$ status at birth with birth weight, length, and head circumference, ${ }^{52,55}$ suggesting these data have robust biological significance.

Our evidence that birth weight and body size increase as CV tHcy concentration is raised while CV $\mathrm{Cbl}$ concentration is decreased would be compatible with an increased utilization of $\mathrm{Cbl}$ in the larger babies as a cofactor required to remethylate Hcy to methionine (Figure 1), perhaps to meet methylation demand ${ }^{1,49}$ and the greater rates of methionine transmethylation in late pregnancy. ${ }^{56} \mathrm{~A}$ higher $\mathrm{CV}$ tHcy concentration, reflecting greater tHcy delivery to the fetus, may also afford the developing fetus an opportunity to metabolize Hcy to methionine. Consistent with this concept, fetal uptake of Hcy is implicated by the lower CA tHcy concentration compared to $\mathrm{CV}$ tHcy, which accords with the previous observations. ${ }^{16,17}$ Additionally, the demonstration that 
methionine synthase activity is present in fetal liver and kidney ${ }^{57}$ lends support to the notion that the fetus has the capacity to remethylate Hcy.

In summary, in normal pregnancy, umbilical plasma tHcy concentration, as a metabolite marker of the functioning of the methionine cycle in the fetal compartment, demonstrated a strong dependence on fetal Cbl, but not folate, concentration. The CPA distensibility, birth weight, and neonatal body size all demonstrated positive associations with fetal tHcy concentration, consistent with the concept of low fetoplacental vascular resistance promoting fetal growth. Further, the inverse associations of neonatal body size indices with fetal $\mathrm{Cb}$ concentration implicate a key modulatory role for $\mathrm{Cbl}$ in regulating fetal Hcy metabolism and fetal growth, which should motivate intervention studies with $\mathrm{Cbl}$ in pregnancy. 


\section{Figures:}

\section{FOLATE CYCLE METHIONINE CYCLE}

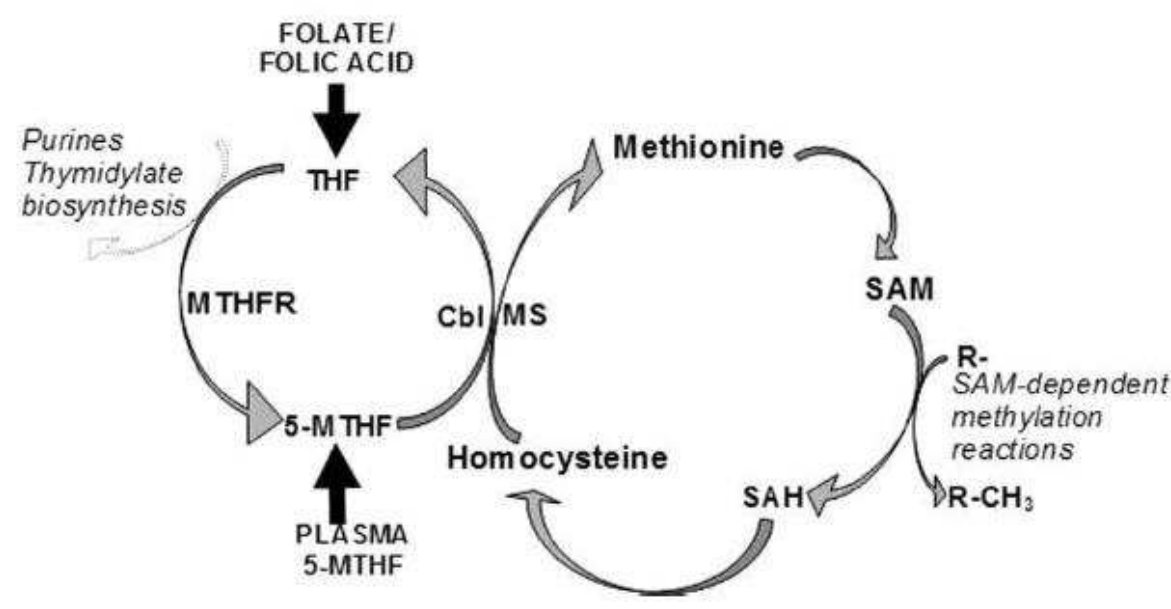

Figure 1. Metabolic coupling of folate and methionine cycles. The conversion of homocysteine (Hcy) to methionine is crucial in generating the methyl donor S-adenosylmethionine (SAM) for methylation processes. Methyl donation from SAM to cellular acceptors (R-) generates S-adenosylhomocysteine (SAH). The efficient metabolism of SAH (by SAH hydrolase catalysis to produce adenosine and Hcy) is essential as SAH inhibits the activity of SAM-dependent methyltransferases involved in a variety of methylation reactions. The physiological form of folate, 5-methyltetrahydrofolate (5-MTHF) serves as the methyl donor in the remethylation of $\mathrm{Hcy}$ to methionine, catalyzed by methionine synthase (MS), a cobalamin (Cbl)dependent enzyme. Methionine synthase is the only enzyme to utilize 5-MTHF and return tetrahydrofolate (THF) to the active folate pool for the de novo synthesis of purines and thymidylate for DNA synthesis. Tetrahydrofolate is then recycled to form 5,10-methylene tetrahydrofolate from which 5-MTHF is generated by the action of 5,10-methylene tetrahydrofolate reductase (MTHFR). 

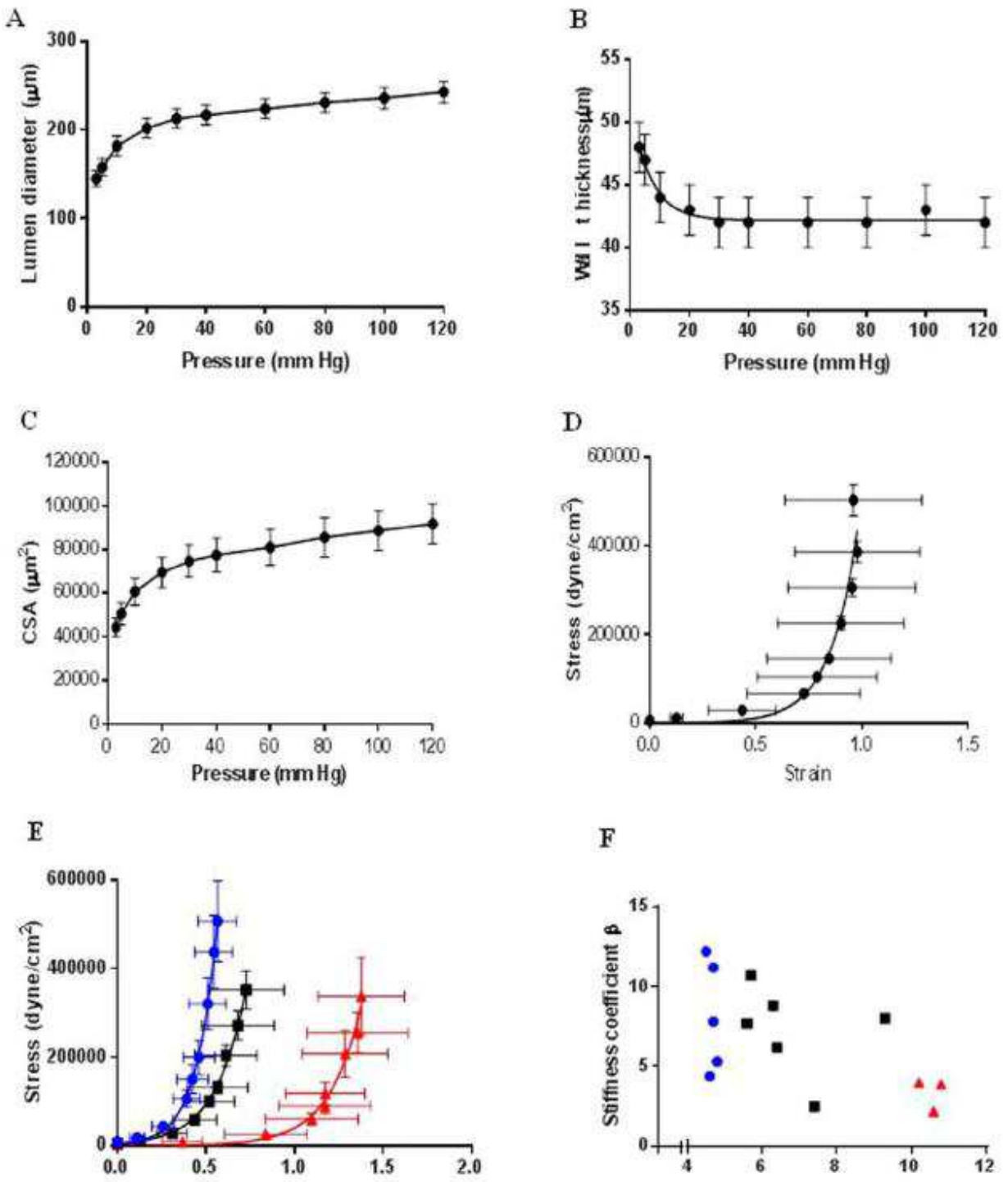

Figure 2. Relationship of the distensibility of placental chorionic plate arteries (CPAs) to umbilical arterial plasma total homocysteine (tHcy) concentration. Pressure myography was applied to human placental CPAs to investigate vascular distensibility changes in (A) vessel lumen diameter, (B) vessel wall thickness, and (C) vessel wall cross-sectional area (CSA) monitored in response to incremental changes in intraluminal pressure. This allowed calculation of (D) the stress-strain relationship, which gives an indication of the passive distensibility of human placental CPAs (for A-D, mean + standard error of the mean [SEM]; $n=34-38$ ). E, Stress-strain relationships in CPAs stratified according to quartile concentrations of cord arterial (CA) tHcy $(\bullet,<25$ th $[n=$ 5]; $\boldsymbol{\square}, 25-75$ th [ $n=6$ ]; $\mathbf{\Lambda},>75$ th [ $n=3$ ]; mean + standard error of the mean [SEM]). $F, \beta$, calculated from the gradient of the stress-strain relationship and a coefficient of vessel stiffness, was inversely correlated with CA plasma tHcy concentration ( $r=-.62, P=.02, n=14$; Spearman rank correlation). Values of $\beta$ are shown according to the quartile concentrations of cord arterial tHcy $(\bullet,<25$ th $[n=5] ; \mathbf{\square}, 25-75$ th $[n=6] ; \mathbf{\Lambda},>75$ th [n = 3]). 


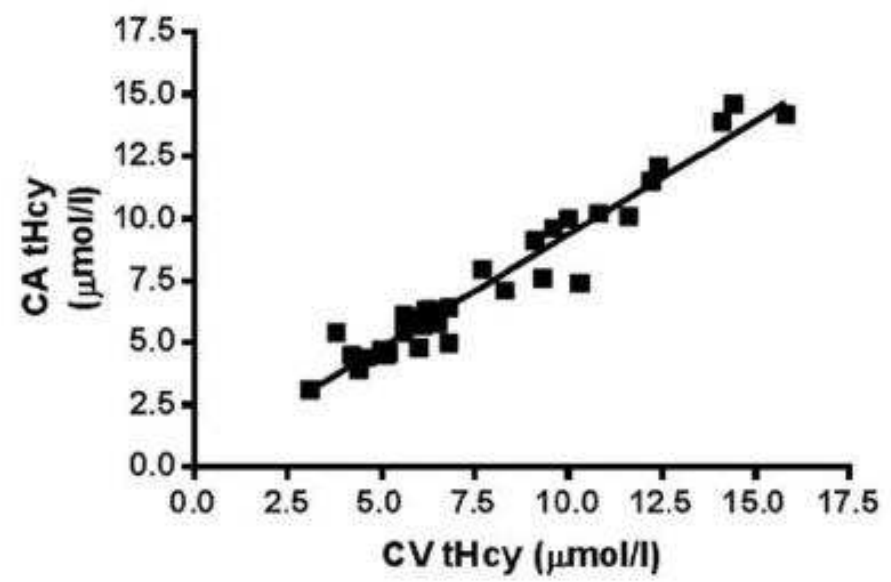

Figure 3. Relationship between plasma total homocysteine (tHcy) concentrations in paired samples of cord venous (CV) and cord arterial (CA) plasma. The concentration of tHcy was measured in paired samples of $\mathrm{CV}$ and CA plasma harvested from the umbilical cord of placentas from normal, uncomplicated pregnancies. There was a linear association between the concentration of tHcy in CV and CA circulations $\left(r^{2}=.94, P<.0001, n=\right.$ 33).

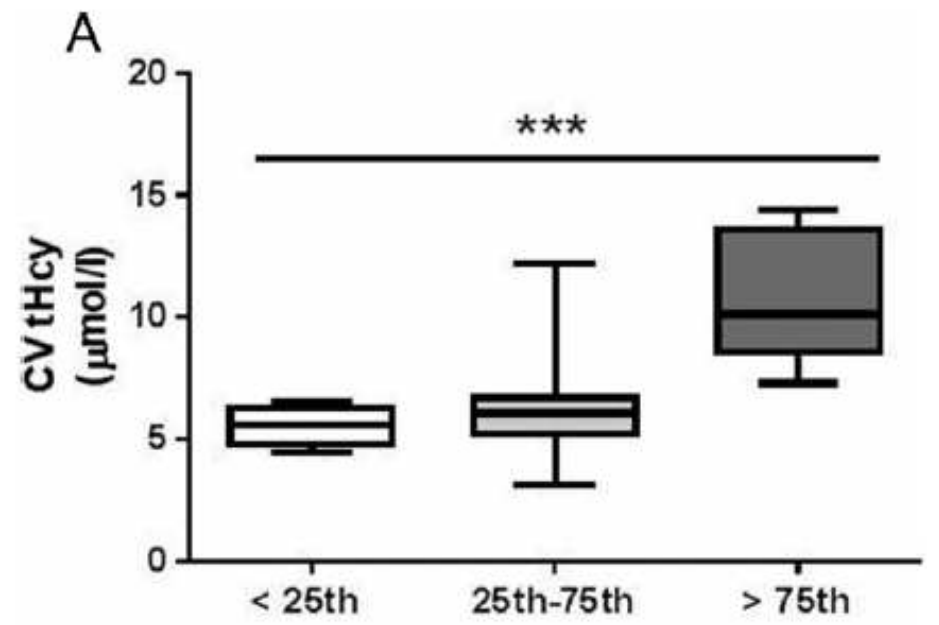

Corrected birthweight centile

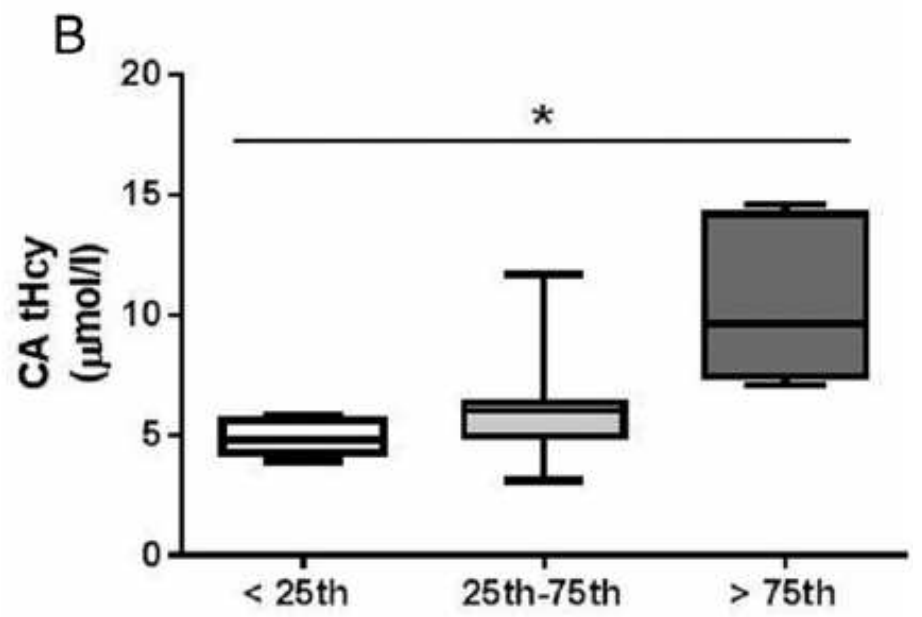

Corrected birthweight centile 
Figure 4. Relationship of corrected birth weight percentile to fetal plasma total homocysteine (tHcy) concentration in paired cord venous (CV) and cord arterial (CA) blood samples. Relationship of corrected birth weight percentile (birth weights adjusted for maternal height, weight, ethnicity and parity, infant sex, and gestational age) stratified according to $<25$ th $(n=5)$, 25th to 75th $(n=12)$, and $>75$ th $(n=8)$ percentiles to (A) CV plasma tHcy concentration and (B) CA plasma tHcy concentration. ${ }^{*} \mathrm{P}<.05$, corrected birth weight percentile versus $\mathrm{CA}$ tHcy; ${ }^{* * *} \mathrm{P}<.005$, corrected birth weight percentile versus $\mathrm{CV}$ tHcy (Kruskal-Wallis test).

\section{$\underline{\text { Tables }}$}

Table 1. Maternal and Newborn Infant Characteristics

\begin{tabular}{lll}
\hline & $\mathrm{n}$ & Median (quartiles) \\
\hline Mothers & & \\
Age, years & 49 & $27(23,32)$ \\
Parity & & \\
1 & 25 & \\
2 & 14 & \\
3 or more & 10 & \\
Height, cm & 49 & $166(163,171)$ \\
Weight, kg & 49 & $67(58,78)$ \\
BMI & 49 & $24(22,27)$ \\
Mode of delivery & & \\
Vaginal & 34 & \\
Cesarean delivery & 15 & \\
Blood pressure, mm Hg & & \\
Systolic & 49 & $108(100,115)$ \\
Diastolic & 49 & $64(60,70)$ \\
Newborn infants & & \\
Gestational age, weeks & 49 & $40(39,41)$ \\
Boys & 21 & \\
Girls & 28 & \\
Birth weight, kg & 49 & $3.64(3.25,4.02)$ \\
$<25$ & 11 & $3.10(2.78,3.31)$ \\
$25-75$ & 25 & $3.64(3.33,3.75)$ \\
$>75$ & 13 & $4.26(3.99,4.48)$ \\
Length, cm & 49 & $51.3(50.1,53.5)$ \\
Mid-arm circumference, cm & 49 & $11.2(10.6,12.1)$ \\
Occipitofrontal circumference, cm & 49 & $35.4(34.5,36.0)$ \\
Blood pressure, mm Hg & & \\
Systolic & 49 & $78(71,85)$ \\
Diastolic & 49 & $43(36,49)$ \\
Mean & 49 & $53(47,62)$ \\
Heart rate per minute & 49 & $131(121,142)$ \\
Cord venous blood & & \\
& & \\
\hline
\end{tabular}




\begin{tabular}{|lll|}
\hline Plasma tHcy, $\mathrm{mmol} / \mathrm{L}$ & 38 & $6.25(5.10,9.95)$ \\
Plasma folate, $\mathrm{ng} / \mathrm{mL}$ & 27 & $22.05(14.20,35.35)$ \\
Plasma Cbl, ng/L & 28 & $348(266,377)$ \\
Cord arterial blood & & \\
Plasma tHcy, $\mathrm{mmol} / \mathrm{L}$ & 29 & $6.30(5.10,10.45)$ \\
Plasma folate, $\mathrm{ng} / \mathrm{mL}$ & 14 & $17.45(12.45,31.85)$ \\
Plasma Cbl (ng/L) & 14 & $284(225,352)$ \\
& & \\
\end{tabular}

Abbreviations: BMI, body mass index; Cbl, cobalamin; tHcy, total homocysteine.

${ }^{a}$ Corrected birth weight percentile: birth weight was adjusted for maternal height, weight, ethnicity, and parity, infant sex, and gestational age.

Table 2. Relationships Between Plasma tHcy, Folate, and Cbl Concentrations in CV Plasma and Birt Weight and Anthropometric Measurements in Newborns.

\begin{tabular}{clll} 
& \multicolumn{3}{c}{ CV Plasma } \\
\cline { 2 - 4 } Infant Size & $\mathrm{tHcy}, \mathrm{mmol} / \mathrm{L}$ & Folate, $\mathrm{ng} / \mathrm{mL}$ & $\mathrm{Cbl}, \mathrm{ng} / \mathrm{L}$ \\
\hline Birth weight, $\mathrm{kg}$ & & & \\
$\mathrm{r}(\mathrm{n})$ & $.67(36)$ & $-0.01(27)$ & $-0.43(28)$ \\
$\mathrm{P}$ & $<.0001^{\mathrm{a}}$ & .96 & $.022^{\mathrm{a}}$
\end{tabular}

Corrected birth weight percentile

$\begin{array}{llll}r(n) & -.59(36) & -.05(27) & -.51(28) \\ P & .0001^{\mathrm{a}} & .81 & .005^{\mathrm{a}}\end{array}$

Length, $\mathrm{cm}$

$\begin{array}{llll}r(n) & .48(36) & .26(27) & -.43(28) \\ P & .003^{\mathrm{a}} & .19 & .022^{\mathrm{a}}\end{array}$

Occipitofrontal head circumference, $\mathrm{cm}$

$\begin{array}{llll}r(n) & .43(36) & -.15(27) & -.29(28) \\ \mathrm{P} & .0087^{\mathrm{a}} & .46 & .14\end{array}$

Mid-arm circumference, $\mathrm{cm}$
$r(n)$
$.47(36)$
$.004^{\mathrm{a}}$
$.05(27)$
$-.55(28)$
.81
$.0024^{\mathrm{a}}$

Abbreviations: Cbl, cobalamin; CV, cord venous; $r(n)$ : Spearman correlation (number of newborn infants); tHcy, total homocysteine.

aDenotes statistical significance. 


\section{References}

1. Solanky N, Requena Jimenez A, D'Souza SW, Sibley CP, Glazier JD. Expression of folate transporters in human placenta and implications for homocysteine metabolism. Placenta. 2010;31(2): 134143.

2. Refsum $\mathrm{H}$. Folate, vitamin B12 and homocysteine in relation to birth defects and pregnancy outcome. Br J Nutr. 2001;85 (suppl 2):S109-S113.

3. Selhub J. The many facets of hyperhomocysteinemia: studies from the Framingham cohorts. J Nutr. 2006;136(6 suppl): 1726S-1730S.

4. Refsum $H$, Ueland PM, Nygård O, Vollset SE. Homocysteine and cardiovascular disease. Ann Rev Med. 1998;49:31-62.

5. Li F, Watkins D, Rosenblatt DS. Vitamin B(12) and birth defects. Mol Genet Metab. 2009;98(1-

2):166-172.

6. Ladipo OA. Nutrition in pregnancy: mineral and vitamin supplements. Am J Clin Nutr. 2000;72 (1 suppl): 280S-290S.

7. Ray JG, Laskin CA. Folic acid and homocyst(e)ine metabolic defects and the risk of placental abruption, pre-eclampsia and spontaneous pregnancy loss: a systematic review. Placenta. 1999;20(7):519-529.

8. Vollset SE, Refsum H, Irgens LM, et al. Plasma total homocysteine, pregnancy complications, and adverse pregnancy outcomes: the Hordaland Homocysteine study. Am J Clin Nutr. 2000;71(4):962-968.

9. Refsum H, Nurk E, Smith AD, et al. The Hordaland Homocysteine Study: a community-based study of homocysteine, its determinants, and associations with disease. J Nutr. 2006;136 (6 suppl): 1731S1740 S.

10. Murphy MM, Fernandez-Ballart JD. Homocysteine in pregnancy. Adv Clin Chem. 2011;53:105137.

11. Hogeveen M, Blom HJ, den Heijer M. Maternal homocysteine and small-for-gestational age offspring: systemic review and meta-analysis. Am J Clin Nutr. 2012;95(1):130-136.

12. Hogeveen $\mathrm{M}$, Blom $\mathrm{HJ}$, van der Heijden $\mathrm{EH}$, et al. Maternal homocysteine and related $\mathrm{B}$ vitamins as risk factors for low birth-weight. Am J Obstet Gynecol. 2010;202(6):572.e1-572.e6.

13. Takimoto $\mathrm{H}$, Mito N, Umegaki K, et al. Relationship between dietary folate intakes, maternal plasma total homocysteine and B-vitamins during pregnancy and fetal growth in Japan. Eur J Nutr. 2007;46(5):300-306. 
14. Murphy MM, Scott JM, Arija V, Molloy AM, Fernandez-Ballart JD. Maternal homocysteine before conception and throughout pregnancy predicts fetal homocysteine and birthweight. Clin Chem. 2004;50(8):1406-1412.

15. Ronnenberg AG, Goldman MB, Chen D, et al. Preconception homocysteine and B vitamin status and birth outcomes in Chinese women. Am J Clin Nutr. 2002;76(6):1385-1391.

16. Raijmakers MT, Roes EM, Steegers EA, van Der Wildt B, PetersWH. Umbilical cord and maternal plasma thiol concentrations in normal pregnancy. Clin Chem. 2001;47(4):749-751.

17. Malinow MR, Rajkovic A, Duell PB, Hess DL, Upson BM. The relationship between maternal and neonatal umbilical cord plasma homocyst(e)ine suggests a potential role for maternal homocyst(e)ine in fetal metabolism. Am J Obstet Gynecol. 1998;178(2):228-233.

18. Infante-Rivard C, Rivard GE, Gauthier R, The'orêt Y. Unexpected relationship between plasma homocysteine and intrauterine growth restriction. Clin Chem. 2003;49(9):1476-1482.

19. Guthikonda S, Haynes WG. Homocysteine: role and implications in atherosclerosis. Curr Atheroscler Rep. 2006;8(2):100-106.

20. Wald DS, Law M, Morris JK. Homocysteine and cardiovascular disease: evidence on causality from a meta-analysis. Br Med J. 2002;325(7374):1202.

21. Woo KS, Chook P, Lolin YI, et al. Hyperhomocyst(e)inemia is a risk factor for arterial endothelial dysfunction in humans. Circulation. 1997;96(8):2542-2544.

22. Chambers JC, Obeid OA, Kooner JS. Physiological increments in plasma homocysteine induce vascular endothelial dysfunction in normal human subjects. Arterioscler Thromb Vasc Biol. 1999; 19(12):2922-2927.

23. Moat SJ, Lang D, McDowell IF, et al. Folate, homocysteine, endothelial function and cardiovascular disease. J Nutr Biochem. 2004;15(2):64-79.

24. Tsai JC, Perrella MA, Yoshizumi M, et al. Promotion of vascular smooth muscle cell growth by homocysteine: a link to atherosclerosis. Proc Natl Acad Sci U S A. 1994;91(14): 63696373.

25. Majors A, Ehrhart LA, Pezacka EH. Homocysteine as a risk factor for vascular disease. Enhanced collagen production and accumulation by smooth muscle cells. Arterioscler Thromb Vasc Biol. 1997;17(10):2074-2081.

26. Majors AK, Sengupta S, Jacobsen DW, Pyeritz RE. Upregulation of smooth muscle cell collagen production by homocysteine insight into the pathogenesis of homocystinuria. Mol Genet Metab. 2002;76(2):92-99.

27. Charpiot $P$, Bescond $A$, Augier $T$, et al. Hyperhomocysteinemia induces elastolysis in minipig arteries: structural consequences, arterial site specificity and effect of captoprilhydrochlorothiazide. Matrix Biol. 1998;17(8-9):559-574.

28. Wareing M, Crocker IP, Warren AY, Taggart MJ, Baker PN. Characterization of small arteries isolated from the human placental chorionic plate. Placenta. 2002;23(5):400-409. 
29. Sweeney M, Wareing M, Mills TA, Baker PN, Taggart MJ. Characterisation of tone oscillations in placental and myometrial arteries from normal pregnancies and those complicated by pre-eclampsia and growth restriction. Placenta. 2008;29(4):356-365

30. Wareing M. Effects of oxygenation and luminal flow on human placenta chorionic plate blood vessel function J Obstet Gynaecol Res. 2012;38(1):185-19 1.1078 Reproductive Sciences 24(7)

31. Kent AL, Kecskes Z, Shadbolt B, Falk MC. Normative blood pressure data in the early neonatal period. Pediatr Nephrol. 2007;22(9):1335-1341.

32. Moat SJ, Bonham JR, Tanner MS, Allen JC, Powers HJ. Recommended approaches for the laboratory measurement of homocysteine in the diagnosis and monitoring of patients with hyperhomocysteinemia. Ann Clin Biochem. 1999;36(pt 3): 372-379.

33. Hausman N, Martin J, Taggart MJ, Austin C. Age-related changes in the contractile and passive arterial properties of murine mesenteric small arteries are altered by caveolin-1 knockout. J Cell Mol Med. 2012;16(8):1720-1730.

34. Gardosi J, Francis A. Customised weight calculator. GROW version 6.5, Year 2011, Gestational Network. www.gestation.net.

35. Braekke K, Ueland PM, Harsem NK, Karlsen A, Blomhoff R,Staff AC. Homocysteine, cysteine and related metabolites in maternal and fetal plasma in preeclampsia. Pediatr Res. 2007; 62(3):319-324.

36. Molloy AM, Mills JL, McPartlin J, Kirke PN, Scott JM, Daly S. Maternal and fetal plasma homocysteine concentrations at birth: the influence of folate, vitamin B12, and the 5,10methylenetetrahydrofolate reductase $677 \mathrm{C} \rightarrow$ T variant. Am J Obstet Gynecol. 2002;186(3):499503.

37. Wareing M, Akef H, Greenwood SL, Bugg G, Baker PN. Umbilical artery Doppler waveform indices from normal pregnant women are related to vasodilatation of placental chorionic plate small arteries. J Obstet Gynaecol. 2005;25(3):248-252.

38. Sweeney M, Jones CJ, Greenwood SL, Baker PN, Taggart MJ. Ultrastructural features of smooth muscle and endothelial cells of isolated isobaric human placental and maternal arteries. Placenta. 2006;27(6-7):635-647.

39. Phillips JK, Vance AM, Raj RS, Mandala' M, Linder EA, Gokina NI. Impact of experimental diabetes on the maternal uterine vascular remodeling during rat pregnancy. Reprod Sci. 2012;19(3): 322-331.

40. Izzard AS, Horton S, Heerkens EH, Shaw L, Heagerty AM. Middle cerebral artery structure and distensibility during developing and established phases of hypertension in the spontaneously hypertensive rat. J. Hypertension. 2006;24(5):875-880.

41. Bund SJ. Spontaneously hypertensive rat resistance artery structure related to myogenic and mechanical properties. Clin Sci. 2001;101(4):385-393.

42. Gonza'lez JM, Briones AM, Starcher B, et al. Influence of elastin on rat small artery mechanical properties. Exp Physiol. 2005; 90(4):463-468. 
43. Symons JD, Zaid UB, Athanassious CN, Mullick AE, Lentz SR, Rutledge JC. Influence of folate on arterial permeability and stiffness in the absence or presence of hyperhomocysteinemia. Arterioscler Throm Vasc Biol. 2006;26(4):814-818.

44. Shargorodsky M, Boaz M, Pasternak S, et al. Serum homocysteine, folate, vitamin $B_{12}$ levels and arterial stiffness in diabetic patients: which of them is really important in atherogenesis? Diabetes Metab Res Rev. 2009;25(1):70-75.

45. Dobrin PB. Mechanical properties of arteries. Physiol Rev. 1978; 58(2):397-460.

46. Overchkin AV, Tyagi N, Sen U, et al. 3-Deazaadenosine mitigates arterial remodelling and hypertension in hyperhomocysteinemic mice. Am J Physiol Lung Cell Mol Physiol. 2006;291(5): L905-L911.

47. Ungvari Z, Koller A. Homocysteine reduces smooth muscle $\left[\mathrm{Ca}^{2 \mathrm{~b}}\right]_{i}$ and constrictor responses of isolated arterioles. J Cardiovasc Pharmacol. 2001;37(6):705-712.

48. Tsitsiou E, Sibley CP, D’Souza SW, Catanescu O, Jacobsen DW, Glazier JD. Homocysteine transport by systems $\mathrm{L}, \mathrm{A}$ and $\mathrm{y}^{\mathrm{b}} \mathrm{L}$ across the microvillous plasma membrane of human placenta. $\mathrm{J}$ Physiol. 2009;587(pt 16):4001-4013.

49. Tsitsiou E, Sibley CP, D'Souza SW, Catanescu O, Jacobsen DW, Glazier JD. Homocysteine is transported by the microvillous plasma membrane of human placenta. J Inherit Metab Dis. 2011;34(1):57-65.

50. Molloy AM, Mills JL, Cox C, et al. Choline and homocysteine interrelations in umbilical cord and maternal plasma at delivery. Am J Clin Nutr. 2005;82(4):836-842.

51. Obeid R, Munz W, Jäger M, Schmidt W, Herrmann W. Biochemical indexes of the B vitamins in cord serum are predicted by maternal B vitamin status. Am J Clin Nutr. 2005;82(1):133-139.

52. Hay G, Clausen T, Whitelaw A, et al. Maternal folate and cobalamin status predicts vitamin status in newborns and 6-month-old infants. J Nutr. 2010;140(3):557-564.

53. Bjørke Monsen AL, Ueland PM, Vollset SE, et al. Determinants of cobalamin status in newborns. Pediatrics. 2001;108(3): 624-630.

54. Murphy MM, Scott JM, McPartlin JM, Fernandez-Ballart JD. The pregnancy-related decrease in fasting plasma homocysteine is not explained by folic acid supplementation, hemodilution, or a decrease in albumin in a longitudinal study. Am J Clin Nutr.2002;76(3):614-619.

55. Obeid R, Morkbak AL, Munz W, Nexo E, Hermann W. The cobalamin-binding proteins transcobalamin and haptocorrin in maternal and cord blood sera at birth. Clin Chem. 2006;52(2): 263-269.

56. Dasarathy J, Gruca LL, Bennett C, et al. Methionine metabolism in human pregnancy. Am J Clin Nutr. 2010;91(2):357-365. 57. Gaull GE, Von Berg W, Räihä NC, Sturman JA. Development of methyltransferase activities of human fetal tissues. Pediatr Res. 1973;7(5):527-533. 